\title{
STABILITY AND LYAPUNOV FUNCTIONS FOR REACTION-DIFFUSION SYSTEMS*
}

\author{
W. B. FITZGIBBON,$\dagger$ S. L. HOLLIS,$\ddagger$ AND J. J. MORGAN $\S$
}

\begin{abstract}
It is shown for a large class of reaction-diffusion systems with Neumann boundary conditions that in the presence of a separable Lyapunov structure, the existence of an a priori $L^{r}$-estimate, uniform in time, for some $r>0$, implies the $L^{\infty}$ uniform stability of steady states. The results are applied to a general class of Lotka-Volterra systems and are seen to provide a partial answer to the global existence question for a large class of balanced systems with nonlinearities that are not bounded by any polynomial.
\end{abstract}

Key words. reaction-diffusion systems, stability, Lyapunov functions

AMS subject classifications. 35B35, 35K45, 35K57

\section{Introduction.}

One of the persistent problems in the theory of systems of reaction diffusion equations concerns the description of the qualitative effects of adding diffusion to systems of ordinary differential equations. To be more precise, if $f=\left(f_{i}\right)_{i=1}^{m}: \mathbb{R}^{m} \rightarrow \mathbb{R}^{m}$, then solutions to the system of ordinary differential equations

$$
\begin{aligned}
\dot{u}(t) & =f(u(t)), \quad t>0, \\
u(0) & =u_{0}
\end{aligned}
$$

determine constant solutions to the reaction-diffusion system

$$
\begin{array}{rlrl}
\partial u / \partial t & =D \Delta u+f(u) & & \text { on } \Omega \times(0, \infty), \\
\partial u / \partial \mathbf{n}=0 & & \text { on } \partial \Omega \times(0, \infty), \\
u(\cdot, 0) & =u_{0}(\cdot) & & \text { on } \Omega,
\end{array}
$$

where $u=\left(u_{1}, \ldots, u_{m}\right)^{T}, D$ is a diagonal matrix with distinct entries $d_{i}>0$ along the diagonal and $\Delta$ denotes the vector Laplacian. One principal question associated with these systems is whether or not global existence of solutions to (1.1) for all choices of initial data guarantees global existence of solutions to (1.2) for all choices of sufficiently smooth initial data. This question had remained unresolved until recent work of Pierre and Schmidt [19]. In that work, the authors give an example of a two component system for which solutions

*Received by the editors August 1, 1994; accepted for publication (in revised form) January 16, 1996.

$\dagger$ Department of Mathematics, University of Houston, Houston, Texas 77204 (fitz@math.uh.edu). The research of this author was supported in part by National Science Foundation grant DMS-9207064.

$\ddagger$ Department of Mathematics and Computer Science, Armstrong Atlantic State University, Savannah, GA 31419 (selwyn_hollis@mailgate.armstrong.edu).

$\S$ Department of Mathematics, Texas A\&M University, College Station, Texas 77843 (jmorgan@ math.tamu.edu). The research of this author was supported in part by National Science Foundation grant DMS-9208046. 
to (1.1) exist globally, while those to the partial differential equation blow up in finite time. Their work is related to a long standing question pointed out by R. H. Martin in the early 1980s regarding the global existence of nonnegative solutions to two component systems of the form

$$
\begin{aligned}
& \partial u / \partial t=d_{1} \Delta u+f_{1}(u, v) \\
& \partial v / \partial t=d_{2} \Delta v+f_{2}(u, v)
\end{aligned} \quad \text { on } \Omega \times(0, \infty)
$$

where $f_{1}(0, v), f_{2}(u, 0) \geq 0$ for all $u, v \geq 0$ and $f_{1}(u, v)+f_{2}(u, v) \leq 0$ for all $u, v \geq 0$. These two conditions on the vector field $\left(f_{1}, f_{2}\right)$ are referred to respectively as quasipositivity and balancing.

This has given rise to similar questions about more general, balanced, quasipositive reaction-diffusion systems of the form

$$
\partial u_{i} / \partial t=d_{i} \Delta u_{i}+f_{i}(u) \quad \text { on } \Omega \times(0, \infty), i=1, \ldots, m
$$

where $u=\left(u_{1}, \ldots, u_{m}\right)^{T}, f_{i}(u) \geq 0$ whenever $u \in \mathbb{R}_{+}^{m}$ with $u_{i}=0$, and $\sum_{i=1}^{m} f_{i}(u) \leq 0$ for all $u \in \mathbb{R}_{+}^{m}$. It should be noted that these balancing and quasipositivity assumptions easily imply that $f(0)=0$ and that all solutions of the ordinary differential equation in (1.1) having nonnegative initial data exist and are bounded for all $t \geq 0$. In particular, for any $M>0$ the region

$$
\left\{u \mid \sum_{i=1}^{n} u_{i} \leq M, \quad u_{i} \geq 0\right\}
$$

is invariant for these systems; therefore the zero solution is stable with respect to $\mathbb{R}_{+}^{m}$.

This structure is merely a simple case of a more general, separable Lyapunov structure for (1.1). Such a structure has the form $H(u)=\sum_{i=1}^{m} h_{i}\left(u_{i}\right)$ where $H: \mathbb{R}_{+}^{m} \rightarrow[0, \infty)$ is a convex function that has a unique zero in $\mathbb{R}_{+}^{m}$ and whose level hypersurfaces bound invariant regions for solutions of (1.1). The existence of such an $H$ easily guarantees the stability of the steady state $z$. Recent work in this vein includes [1], [5], [6], [10], [14], and [16].

The work at hand concerns the persistence of stability of steady-state solutions to (1.1), in the presence of a separable Lyapunov structure, when diffusion is added to the system; that is, in the setting of (1.2) with nonnegative, continuous initial data. Questions of stability for nonlinear systems are frequently resolved via linearized stability or Lyapunov type methods. Typically when one attempts to lift Lyapunov functions from the setting of (1.1) to (1.2), one obtains estimates in $L_{1}(\Omega)$ or $L_{p}(\Omega)$ and not the optimal uniform $L_{\infty}(\Omega)$ estimates needed to obtain stability. Therefore, the central theme of our work will be the introduction of an intermediate notion of stability from $C(\bar{\Omega})$ to $L_{p}(\Omega)$, and the bootstrapping of $L_{p}(\Omega)$ estimates to $L_{\infty}(\Omega)$.

We should point out the phenomena of diffusion driven instabilities. It is well-known that the addition of diffusion can destabilize constant steady-states; see, e.g., [18]. Therefore, we shall be lead to the conclusion that the systems of ordinary differential equations which admit diffusion driven instability do not have a Lyapunov structure of the type to be described. 
Our subsequent development consists of five sections. In addition to detailing our hypotheses and outlining relevant theory, the second section introduces the central notion of stability from $C(\bar{\Omega})$ to $L_{p}(\Omega)$ and bootstraps this stability from $L_{p}(\Omega)$ to $L_{\infty}(\Omega)$. As such, the second section forms the theoretical basis of the paper. The third section introduces the notion of $D$-diffusively convex Lyapunov functionals and demonstrates the connection to the work in Section 2. The fourth section is concerned with application of the theory. It begins by considering balanced two-component systems and then applies the theory to dissipative chemical systems and Lotka-Volterra systems. We conclude with some general comments and remarks.

We conclude this section with two remarks. First, our result gives a partial answer to R.H. Martin's original question. We determine that balanced, quasipositive reactiondiffusion systems subject to homogeneous Neumann boundary conditions have global solutions for all choices of continuous, sufficiently small, nonnegative initial data. Second, we have limited our discussion to the case of homogeneous Neumann boundary conditions for the following reason. If all components of our system satisfy strictly dissipative boundary conditions, such as homogeneous Dirichlet or homogeneous Robin, then the presence of a separable Lyapunov structure along with these boundary conditions allows one to employ linearized stability arguments to obtain asymptotic stability. In the case of a mixture of homogeneous Neumann boundary conditions and dissipative conditions (as mentioned above) the arguments follow our development.

\section{Preliminaries and $\infty-r$ stability.}

In what follows, $\Omega$ shall be a bounded domain in $\mathbb{R}^{n}$ that lies locally on one side of its $C^{2+\alpha}$ boundary $\partial \Omega$. We shall always assume that the initial data $u_{0}=\left(u_{0_{1}}, \ldots, u_{0_{m}}\right)^{T} \in$ $C(\bar{\Omega})^{m}$ and that the vector field $f=\left(f_{i}\right)_{i=1}^{m}$ has the property that

$$
f \in C^{1}\left(\mathbb{R}^{m} ; \mathbb{R}^{m}\right) .
$$

However, we make no assumptions concerning the growth rates of the individual components $f_{i}$ of $f$. The symbol $D$ will denote an $m \times m$ diagonal matrix with distinct entries $d_{i}>0, i=1$ to $m$, along the diagonal. We point out that all results contained herein would trivialize, were we to assume that the $d_{i}$ s were identical. We hope that we shall not introduce undue confusion by using the symbol " $\Delta$ " to denote both the vector and the scalar Laplacian. Equations without subscripts will typically denote vector equations and nonsubscripted scalar equations shall be specifically referred to as such.

In our general discussion we use the notation $z_{0}=\left(z_{0_{1}}, \ldots, z_{0_{m}}\right)^{T} \in \mathbb{R}^{m}$ to denote an equilibrium point (or steady state) of (1.1). Namely, we have

$$
f\left(z_{0}\right)=0 \text {. }
$$

A closed subset of $M \subseteq \mathbb{R}^{m}$ will be called a forward invariant set for (1.2) if $u_{0}(x)=$ $\left(u_{0_{1}}(x), \ldots, u_{0_{m}}(x)\right)^{T} \in M$ for all $x \in \Omega$ implies that

$$
u(x, t)=\left(u_{1}(x, t), \ldots, u_{m}(x, t)\right) \in M
$$

for all $(x, t) \in \Omega \times\left[0, T_{\max }\right)$. Here, $\left[0, T_{\max }\right)$ denotes the maximal interval of existence for solutions to the initial boundary value problem (1.2). We shall require that there exists a 
forward invariant set $M$ (not necessarily bounded) for solutions to (1.2). Hence, because the $d_{i}$ are assumed to be distinct, we assume that there exists a forward invariant $m$-cube

$$
M=M_{1} \times \cdots \times M_{m}
$$

for (1.2), where each $M_{i}, i=1$ to $m$, is a closed interval. We point out that we have said nothing concerning the boundedness of $M$ and consequently we make no presuppositions concerning the global existence of solutions to (1.2). For example, $M$ may well be $\mathbb{R}_{+}^{m}$ (the positive orthant) or all of $\mathbb{R}^{m}$.

In what follows, the mild abuse of notation $v \in M$ will be used frequently to indicate that a function $v: \Omega \rightarrow \mathbb{R}^{m}$ has the property that $v(x) \in M$ for all $x \in \Omega$.

Our analysis will involve the standard Lebesgue spaces $L_{p}(\Omega), p \geq 1$ :

$$
\begin{aligned}
L_{p}(\Omega) & =\left\{\left.u\left|\int_{\Omega}\right| u\right|^{p} d x<\infty\right\} ; \\
\|u\|_{p, \Omega} & =\left(\int_{\Omega}|u|^{p} d x\right)^{1 / p} .
\end{aligned}
$$

We shall also want to consider the analogous spaces obtained with $0<p<1$. Although (2.6) does not define a norm on $L_{p}(\Omega)$ if $0<p<1$, we will use the same notation for the functional defined on $L_{p}(\Omega)$ by the right side of (2.6). If $p \geq 1$ and $k>0$ then $W_{p}^{(k)}(\Omega)$ denotes the usual $k^{\text {th }}$ order Sobolev space in $L_{p}(\Omega)$ and $W_{p}^{(2 k, k)}(\Omega \times(\tau, T))$ denotes its analogue in $L_{p}(\Omega \times(\tau, T))$. For definitions of these spaces for both integral and nonintegral $k$, we refer the reader to [12].

We will need the following fractional Sobolev space embedding theorem of Amann [2].

THEOREM 2.7. Let $k \in \mathbb{N}$ and suppose that $\partial \Omega$ is uniformly regular of class $C^{k}$. If $0 \leq s^{\prime} \leq s \leq k$ and $1<p, q<\infty$ then $W_{p}^{s}(\Omega)$ embeds continuously in $W_{q}^{s^{\prime}}(\Omega)$ whenever $1 / p \geq 1 / q$ and $s-(n / p) \geq s^{\prime}-(n / q)$.

We now introduce the notion of $\infty-r$ stability. It will be a notion of stability with respect to $M$, which will allow us to consider steady states belonging to $\partial M$.

DeFINITION 2.8. Let $z_{0} \in M$ be an equilibrium point of the vector field $f=\left(f_{i}\right)_{i=1}^{m}$ and let $0<r \leq \infty$. Then $z_{0}$ is said to be uniformly $\infty-r$ stable with respect to $M$ if for all $\varepsilon>0$ there exists a $\delta>0$ such that $u_{0} \in M$ and $\left\|u_{0_{i}}-z_{0_{i}}\right\|_{\infty, \Omega}<\delta$ for $i=1$ to $m$ imply

(i) a classical solution to (1.2) exists on $\Omega \times[0, \infty)$;

(ii) $\left\|u_{0_{i}}(\cdot, t)-z_{0_{i}}\right\|_{r, \Omega}<\varepsilon$ for $i=1$ to $m$ and $t>0$.

An $\infty$-r stable equilibrium point $z_{0} \in M$ is said to be uniformly $\infty-r$ asymptotically stable if there exists a $\delta>0$ such that $u_{0} \in M$ and $\left\|u_{i}-z_{0_{i}}\right\|_{\infty, \Omega}<\delta$ for $i=1$ to $m$ imply

(iii) $\lim _{t \rightarrow \infty}\left\|u_{i}(\cdot, t)-z_{0_{i}}\right\|_{r, \Omega}=0$ for $i=1$ to $m$.

The usual notions of stability with respect to $M$ now correspond to $\infty$ - $\infty$ stability with respect to $M$ as stated formally in the following definition.

Definition 2.9. An equilibrium point $z_{0} \in M$ is said to be uniformly stable with respect to $M$ if it is uniformly $\infty-\infty$ stable with respect to $M$. A stable equilibrium point $z_{0} \in M$ is said to be uniformly asymptotically stable with respect to $M$ if it is uniformly $\infty-\infty$ asymptotically stable with respect to $M$. 
We shall see in the sequel that the notion of $\infty-r$ stability is intermediate and may be subsumed by the notion of stability. For a given point $v_{0} \in \mathbb{R}^{m}$, let the symbol $C_{\eta}\left(v_{0}\right)$ denote the $m$-dimensional cube centered at $v_{0}$ with diameter $2 \sqrt{m} \eta$ and $B_{\delta}\left(v_{0}\right)$ the $m$ dimensional ball of radius $\delta$ about $v_{0}$. We remark that $C_{\varepsilon}\left(v_{0}\right) \subseteq B_{\sqrt{m}_{\varepsilon}}\left(v_{0}\right)$. The analysis that follows will require "cutoff" functions $\varphi_{\eta, v_{0}} \in C^{\infty}\left(\mathbb{R}^{m} ;[0,1]\right)$, defined for $\eta>0$ by

$$
\begin{array}{ll}
\varphi_{\eta, v_{0}}(u)=1 & \text { for } \quad u \in C_{\eta}\left(v_{0}\right) \\
\varphi_{\eta, v_{0}}(u)=0 & \text { for } \quad u \in \mathbb{R}^{m} \backslash C_{2 \eta}\left(v_{0}\right) .
\end{array}
$$

If $z_{0} \in M$ is an equilibrium point we truncate the vector field $f$ by componentwise multiplication by $\varphi_{\eta, z_{0}}$ for $\eta>0$; i.e., we define $f\left[\eta, z_{0}\right]=\left(f_{i}\left[\eta, z_{0}\right]\right)_{i=1}^{m}$ by

$$
f_{i}\left[\eta, z_{0}\right](u)=\varphi_{\eta, z_{0}}(u) f_{i}(u) .
$$

Then solutions to the truncated system

$$
\begin{aligned}
\partial v / \partial t & =D \Delta v+f\left[\eta, z_{0}\right](v) & & \text { on } \Omega \times(0, \infty), \\
\partial v / \partial \mathbf{n} & =0 & & \text { on } \partial \Omega \times(0, \infty), \\
v(\cdot, 0) & =v_{0} & & \text { on } \Omega,
\end{aligned}
$$

where $v_{0_{i}}=\varphi_{\eta, z_{0}}\left(u_{0}\right) u_{0_{i}}$, exist on $\Omega \times[0, \infty)$ and are globally bounded. Moreover, it is trivial to observe that if $z_{0} \in M$ is an equilibrium point of $f$, then $z_{0}$ is also an equilibrium point of $f\left[\eta, z_{0}\right]$.

We now formally state a few simple observations concerning solutions to (2.12).

Lemma 2.13. If $\eta>0, v_{0} \in C\left(\bar{\Omega} ; C_{2 \eta}\left(z_{0}\right)\right)$ and $f\left[\eta, z_{0}\right]$ is the vector field defined via (2.11), then (2.12) has a unique classical solution on $\bar{\Omega} \times[0, \infty)$. Moreover,

(i) $v(\cdot, t) \in C_{2 \eta}\left(z_{0}\right) \cap M$ for $t \geq 0$;

(ii) if $v(\cdot, t) \in C_{\eta}\left(z_{0}\right) \cap M$ for $0 \leq t<T$ then $v(x, t)=u(x, t)$ for $(x, t) \in \Omega \times[0, T)$ where $u$ is the solution to (1.2).

Proof. We observe that $M \cap C_{2 \eta}\left(z_{0}\right)$ is a bounded invariant region for (2.12) because the vector field $f\left[\eta, z_{0}\right]$ is identically zero exterior to $C_{2 \eta}\left(z_{0}\right)$ and does not point out of $M$. Therefore solutions to (2.12) exist globally and remain confined to $M \cap C_{2 \eta}\left(z_{0}\right)$ for all time [21]. Classical uniqueness theory for parabolic equations together with the observation that $\left.f\left[\eta, z_{0}\right]\right|_{C_{\eta}\left(z_{0}\right)}=\left.f\right|_{C_{\eta}\left(z_{0}\right)}$ immediately confirms the second assertion.

LEMma 2.14. If $z_{0}$ is uniformly $\infty-r$ stable for (2.12) with respect to $M$ for some $r \in(0, \infty)$, then $z_{0}$ is uniformly $\infty$-p stable for (2.12) with respect to $M$ for all $p \in(0, \infty)$. Analogous results hold for uniform $\infty-r$ asymptotic stability.

Proof. If $0<p<r$, the results follow easily by the Jensen inequality and the convexity of $g(z)=|z|^{r / p}$. If $r<p$, then because $g(z)=\left|z-z_{0_{i}}\right|^{p-r}$ is bounded above by $(2 \sqrt{m} \eta)^{p-r}$ on $C_{2 \eta}\left(z_{0}\right)$, and $v(x, t) \in C_{2 \eta}\left(z_{0}\right)$ for all $(x, t) \in \Omega \times(0, \infty)$, we have

$$
\left|v_{i}-z_{0_{i}}\right|^{p} \leq(2 \sqrt{m} \eta)^{p-r}\left|v_{i}-z_{0_{i}}\right|^{r} \text { on } \Omega \times(0, \infty),
$$

from which the desired results follow. 
The next theorem provides the foundation of our development. It states that $\infty-r$ stability of the truncated system (2.12) guarantees $\infty-\infty$ stability of the original system (1.2).

THEOREM 2.16. Let $z_{0} \in M$ be an equilibrium point of the vector field $f$. If $r>0$ and $z_{0}$ is a uniformly $\infty-r$ stable equilibrium point for (2.12), then $z_{0}$ is a uniformly stable solution for (1.2). Analogous results hold for uniformly $\infty-r$ asymptotic stability.

Proof. We begin by fixing $\eta>0$. If we are able to choose $\delta>0$ so that solutions to (2.12) have the property that $v_{0} \in C_{\delta}\left(z_{0}\right)$ implies that $v(x, t) \in C_{\varepsilon}\left(z_{0}\right)$, where $\varepsilon<\eta$, then solutions to (2.12) and (1.2) coincide. Therefore it will suffice to demonstrate that uniformly $\infty-r$ stable solutions of (2.12) are uniformly stable solutions of (2.12).

By virtue of Lemma 2.14 with $p=2$, we know that there exists a continuous function $\tilde{\rho}_{1}$ with $\tilde{\rho}_{1}(0)=0$ and $\tilde{\rho}_{1}(s)>0$ for $s>0$ such that for $i=1$ to $m$ and $t \in[0, \infty)$

$$
\left\|v_{i}(\cdot, t)-z_{0_{i}}\right\|_{2, \Omega} \leq \tilde{\rho}_{1}\left(\left\|v_{0}-z_{0}\right\|_{\infty, \Omega}\right)
$$

We shall demonstrate via an iteration scheme that there exists a continuous function $\rho$ with $\rho(0)=0$ and $\rho(s)>0$ such that for $i=1$ to $m$ and $t \in[0, \infty)$ we have

$$
\left\|v_{i}(\cdot, t)-z_{0_{i}}\right\|_{\infty, \Omega} \leq \rho\left(\left\|v_{0}-z_{0}\right\|_{\infty, \Omega}\right)
$$

and we shall thereby obtain our desired conclusion. Toward this end we set

$$
w(x, t)=v(x, t)-z_{0}
$$

and multiply the $i^{\text {th }}$ component of $(2.12 \mathrm{a})$ by $w_{i}$ to obtain

$$
w_{i} \partial w_{i} / \partial t-w_{i} d_{i} \Delta w_{i}=w_{i} f_{i}\left[\eta, z_{0}\right](v)
$$

Because $f\left[\eta, z_{0}\right]$ is Lipschitz there exists an $N$ such that integration of $(2.20)$ on the spacetime cylinder $\Omega \times(\tau, T)$ yields

$$
\begin{aligned}
\frac{1}{2}\left\|w_{i}(\cdot, T)\right\|_{2, \Omega}^{2} & +d_{i} \int_{\tau}^{T} \int_{\Omega}\left|\nabla w_{i}\right|^{2} d x d t \\
& \leq \frac{1}{2}\left\|w_{i}(\cdot, \tau)\right\|_{2, \Omega}^{2}+N \sum_{k=1}^{m} \int_{\tau}^{T} \int_{\Omega}\left|w_{i}\right|\left|w_{k}\right| d x d t
\end{aligned}
$$

This implies that if $\tau \geq 0$ and $\tau+1<T<\tau+3$, then

$$
\begin{aligned}
\frac{1}{2}\left\|w_{i}(\cdot, T)\right\|_{2, \Omega}^{2} & +d_{i} \int_{\tau+1}^{T} \int_{\Omega}\left|\nabla w_{i}\right|^{2} d x d t \\
& \leq \frac{1}{2}\left\|w_{i}(\cdot, \tau)\right\|_{2, \Omega}^{2}+N \sum_{k=1}^{m} \max _{[\tau, \tau+3]} \int_{\Omega}\left|w_{i}\right|\left|w_{k}\right| d x
\end{aligned}
$$


After applying Young's inequality and (2.17) to the right side of (2.21) and the mean value theorem for integrals to the $t$-integral on the left side, we construct an increasing sequence $\left\{T_{1, j}\right\}_{j=1}^{\infty}$ with

$$
T_{1,1} \leq 3 \text { and } 1<T_{1, j+1}-T_{1, j}<3 \quad \forall j \in \mathbb{N}
$$

and a continuous function $\rho_{1}$ with $\rho_{1}(0)=0$ and $\rho_{1}(s)>0$ for $s>0$ such that

$$
\left\|w_{i}\left(\cdot, T_{1, j}\right)\right\|_{2, \Omega}^{(1)} \leq \rho_{1}\left(\left\|v_{0}-z_{0}\right\|_{\infty, \Omega}\right) \quad \forall j \in \mathbb{N} .
$$

Now we begin to make use of a well known classical estimate for parabolic initial boundary value problems from Ladyženskaja, Solonnikov, and Uralćeva [12, p. 341]. More specifically, recall that if $1<q<\infty, 0<\tau<T \leq \tau+3, \theta \in L^{q}(\Omega \times(\tau, T)), \phi_{0} \in W_{q}^{2-2 / q}(\Omega)$, and $\phi$ solves

$$
\begin{aligned}
\partial \phi / \partial t & =d_{i} \Delta \phi+\theta & & \text { on } \Omega \times(\tau, T) \\
\partial \phi / \partial \mathbf{n} & =0 & & \text { on } \partial \Omega \times(\tau, T) \\
\phi(\cdot, \tau) & =\phi_{0} & & \text { on } \Omega
\end{aligned}
$$

then there exists $c>0$ such that

$$
\|\phi\|_{q, \Omega \times(\tau, T)}^{(2,1)} \leq c\left[\|\theta\|_{q, \Omega \times(\tau, T)}+\left\|\phi_{0}\right\|_{q, \Omega}^{(2-2 / q)}\right]
$$

where $c$ depends only on $d_{i}$ and $\Omega$. Applying this parabolic regularity estimate with $q=2$, we obtain a constant $c_{1}>0$ such that

$$
\left\|w_{i}\right\|_{2, \Omega \times\left(T_{1, j}, T_{1, j+1}\right)}^{(2,1)} \leq c_{1}\left(\left\|f_{i}\left[\eta, z_{0}\right](v)\right\|_{2, \Omega \times\left(T_{1, j}, T_{1, j+1}\right)}+\left\|w_{i}\left(\cdot, T_{1, j}\right)\right\|_{2, \Omega}^{(1)}\right) .
$$

We now claim that for every $k \in \mathbb{N}$, there exist

(i) a sequence $\left\{T_{k, j}\right\}_{j=1}^{\infty}$ such that $T_{k, 1} \leq k+2$ and $1<T_{k, j+1}-T_{k, j}<3 \forall j \in \mathbb{N}$,

(ii) a constant $c_{k}>0$, and

(iii) a function $\rho_{k} \in C([0, \infty),[0, \infty))$ such that $\rho_{k}(0)=0$ such that for all $j \in \mathbb{N}$, the estimate

$$
\left\|w_{i}\right\|_{q_{k}, \Omega \times\left(T_{k, j}, T_{k, j+1}\right)}^{(2,1)} \leq c_{k}\left(\left\|f_{i}\left[\eta, z_{0}\right](v)\right\|_{q_{k}, \Omega \times\left(T_{k, j}, T_{k, j+1}\right)}+\rho_{k}\left(\left\|v_{0}-z_{0}\right\|_{\infty, \Omega}\right)\right)
$$

is valid with $q_{k}=2((n+2) / n)^{k-1}$.

To establish this claim, we begin by noting that (2.22), (2.23), and (2.26) combine to give the claim for $k=1$. We now proceed by induction on $k$. Suppose that the claim holds for $k=\ell \geq 1$ and consider the case $k=\ell+1$. Since $f_{i}\left[\eta, z_{0}\right]$ is Lipschitz, we can use our hypothesis and Lemma 2.14 with $p=q_{\ell}$ to conclude from (2.27) that there exists a continuous function $\tilde{\rho}_{\ell}$ such that $\tilde{\rho}_{\ell}(0)=0$ and

$$
\left\|w_{i}\right\|_{q_{\ell}, \Omega \times\left(T_{\ell, j}, T_{\ell, j+4}\right)}^{(2,1)} \leq \tilde{\rho}_{\ell}\left(\left\|v_{0}-z_{0}\right\|_{\infty}\right) \quad \forall j \in \mathbb{N}
$$


Note that $T_{\ell, j+4}-T_{\ell, j}>4$. Therefore (2.28) implies the inequalities:

$$
\int_{T_{\ell, j}}^{T_{\ell, j}+1}\left(\left\|w_{i}\right\|_{q_{\ell}, \Omega}^{(2)}\right)^{q_{\ell}} d t, \int_{T_{\ell, j}+2}^{T_{\ell, j}+3}\left(\left\|w_{i}\right\|_{q_{\ell}, \Omega}^{(2)}\right)^{q_{\ell}} d t \leq\left[\tilde{\rho}_{\ell}\left(\left\|v_{0}-z_{0}\right\|_{\infty, \Omega}\right)\right]^{q_{\ell}} .
$$

Consequently, we can construct a sequence $\left\{T_{\ell+1, j}\right\}_{j=1}^{\infty}$ with

$$
T_{\ell, k}<T_{\ell+1,2 k-1}<T_{\ell, k}+1 \text { and } T_{\ell, k}+2<T_{\ell+1,2 k}<T_{\ell, k}+3
$$

such that

$$
\left\|w_{i}\left(\cdot, T_{\ell+1, j}\right)\right\|_{q_{\ell}, \Omega}^{(2)} \leq \tilde{\rho}_{\ell}\left(\left\|v_{0}-z_{0}\right\|_{\infty}\right) \forall j \in \mathbb{N}
$$

We now apply Theorem 2.7 to conclude that $W_{q_{\ell}}^{(2)}(\Omega)$ imbeds continuously into $W_{q \ell+1}^{\left(2-2 / q_{\ell+1}\right)}(\Omega)$. Therefore there exists $\rho_{\ell+1} \in C([0, \infty),[0, \infty))$ such that $\rho_{\ell+1}(0)=0$ and

$$
\left\|w_{i}\left(\cdot, T_{\ell+1, j}\right)\right\|_{q_{\ell+1}, \Omega}^{\left(2-2 / q_{\ell+1}\right)} \leq \rho_{\ell+1}\left(\left\|v_{0}-z_{0}\right\|_{\infty}\right) \quad \forall j \in \mathbb{N}
$$

Now by combining (2.32) with the parabolic regularity estimate in $(2.25)$, we see that our claim is true for $k=\ell+1$, thus establishing the claim for all $k \in \mathbb{N}$.

Now, with $k$ taken such that $q_{k}>\frac{n+2}{2}$ we have from [12] that there exists $C>0$ such that

$$
\|w\|_{\infty, \Omega \times\left(T_{k, j}, T_{k, j+1}\right)} \leq C\|w\|_{q_{k}, \Omega \times\left(T_{k, j}, T_{k, j+1}\right)}^{(1,2)} \forall j \in \mathbb{N} .
$$

Therefore, if we combine this with our claim above, we find that there exists a continuous function $\tilde{\rho}_{k}$ such that $\tilde{\rho}_{k}(0)=0$ and

$$
\|w\|_{\infty, \Omega \times\left(T_{k, j}, T_{k, j+1}\right)} \leq \tilde{\rho}_{k}\left(\left\|v_{0}-z_{0}\right\|_{\infty}\right) \quad \forall j \in \mathbb{N}
$$

But $T_{k, 1} \leq k+2$, so

$$
\|w\|_{\infty, \Omega \times[k+2, \infty)} \leq \tilde{\rho}_{k}\left(\left\|v_{0}-z_{0}\right\|_{\infty}\right)
$$

We now recall that the operator $-d_{i} \Delta$ with homogeneous Neumann boundary conditions generates a nonexpansive analytic semigroup $T_{i}(t)$ on $C(\bar{\Omega})$; see Stewart [22]. So we have

$$
\begin{aligned}
w_{i}(t) & =T_{i}(t)\left(v_{0_{i}}-z_{0_{i}}\right)+\int_{0}^{t} T_{i}(t-s) f_{i}\left[\eta, z_{0}\right](v(\cdot, s)) d s \\
& =T_{i}(t)\left(v_{0_{i}}-z_{0_{i}}\right)+\int_{0}^{t} T_{i}(t-s)\left(f_{i}\left[\eta, z_{0}\right](v(\cdot, s))-f_{i}\left[\eta, z_{0}\right]\left(z_{0}\right)\right) d s
\end{aligned}
$$

which implies that

$$
\left\|w_{i}(t)\right\|_{\infty, \Omega} \leq\left\|v_{0}-z_{0}\right\|_{\infty, \Omega}+\int_{0}^{t} K_{\eta}\|w(\cdot, s)\|_{\infty, \Omega} d s
$$


Therefore, since $\|w(\cdot, t)\|_{\infty, \Omega}=\max _{1 \leq i \leq m}\left\|w_{i}(\cdot, t)\right\|_{\infty, \Omega}$, we have

$$
\|w(\cdot, t)\|_{\infty} \leq e^{K_{\eta} t}\left\|v_{0}-z_{0}\right\|_{\infty} .
$$

Consequently, because of (2.33) we have

$$
\|w\|_{\infty, \Omega \times \mathbb{R}_{+}} \leq \max \left\{e^{K_{\eta}(k+2)}\left\|v_{0}-z_{0}\right\|_{\infty}, \tilde{\rho}_{k}\left(\left\|v_{0}-z_{0}\right\|_{\infty}\right)\right\} .
$$

Finally, since $\eta>0$ is fixed, for any $\varepsilon \in(0, \eta)$ there exists $\delta>0$ such that

$$
\left\|v_{0}-z_{0}\right\|_{\infty}<\delta \text { implies }\left\|v-z_{0}\right\|_{\infty, \Omega \times \mathbb{R}_{+}}=\|w\|_{\infty, \Omega \times \mathbb{R}_{+}}<\varepsilon .
$$

We point out that if $z_{0}$ is not a constant we can modify the preceding arguments as follows. Suppose that $z_{0}=w$ is a smooth function satisfying

$$
\begin{array}{lr}
-D \Delta w=f(w) & \text { on } \Omega \\
\partial w / \partial \mathbf{n}=0 & \text { on } \partial \Omega
\end{array}
$$

In a manner similar to what was done above, the vector field may be truncated in a rectangular neighborhood containing $\{w(x) \mid x \in \Omega\}$. For $\eta>0$, let $b_{1}(\eta, w)$ be an $m$ dimensional cube such that $w \in \operatorname{int} b_{1}(\eta, w)$ with $\eta=\inf _{x \in \Omega} \operatorname{dist}\left(w(x), \partial b_{1}(\eta, w)\right)$, and let $b_{2}(\eta, w)$ denote the $m$-cube concentric to $b_{1}(\eta, w)$ with twice the diameter. We mollify the characteristic function of $b_{1}(\eta, w)$ to produce a nonnegative function $\varphi_{\eta, w}$ such that:

(i) $\varphi_{\eta, w} \in C^{\infty}\left(\mathbb{R}^{m} ;[0,1]\right)$,

(ii) $\varphi_{\eta, w}(u)=1$ if $u \in b_{1}(\eta, w)$,

(iii) $\varphi_{\eta, w}(u)=0$ if $u \in \mathbb{R}^{m} \backslash b_{2}(\eta, w)$,

and thus produce a corresponding truncated system $(c f .(2.11),(2.12))$ :

$$
\begin{aligned}
\partial v / \partial t & =D \Delta v+f[\eta, w](v) & & \text { on } \Omega \times(0, \infty) \\
\partial v / \partial \mathbf{n} & =0 & & \text { on } \partial \Omega \times(0, \infty) \\
v(\cdot, 0) & =\varphi_{\eta, w}\left(u_{0}\right) u_{0} & & \text { on } \Omega .
\end{aligned}
$$

If $\mu=v-w$ and $\eta$ is chosen such that $\eta>\|w\|_{\infty}$, we have

$$
\begin{array}{rlrl}
\partial \mu_{i} / \partial t & =d_{i} \Delta \mu_{i}+f_{i}[\eta, w](v)-f_{i}[\eta, w](w) & & \text { on } \Omega \times(0, \infty) \\
\partial \mu_{i} / \partial \mathbf{n}=0 & & \text { on } \partial \Omega \times(0, \infty) & \\
\mu_{i}(x, 0)=v_{0_{i}}-w_{i} & & \text { on } \Omega .
\end{array}
$$

Then, it is not difficult to establish an analog of Lemma 2.13 and deduce that global solutions to (2.36) exist and that, if they are sufficiently close to $w$, they satisfy (2.36). The following result concludes this section. Its proof is essentially a verbatim repetition of the one given for Theorem 2.16. 
TheOREm 2.37. Let $w \in M$ be a classical, spatially nonhomogeneous solution to the elliptic system (2.35). If $r>0$ and $w$ is a uniformly $\infty-r$ stable steady-state of (2.36), then $w$ is a uniformly stable steady-state solution of (1.2). Analogous results hold for uniformly $\infty-r$ asymptotically stable solutions.

We remark that an interesting reference pertaining to (2.35) is H. Matano [15].

\section{D-Diffusively convex Lyapunov functionals.}

The most common tool for analyzing he local stability of equilibrium points for systems of ordinary differential equations of the form (1.1) is the principle of linearized stability. If all the eigenvalues of the derivative of $f$ at $z_{0}$ have negative real part then $z_{0}$ is locally asymptotically stable. On the other hand, if any of the eigenvalues have positive real part, then the equilibrium point $z_{0}$ is unstable. These ideas carry over to the context of semilinear parabolic equations; see, e.g., [9]. In the case of nonhyperbolic equilibrium points, however, linearization methods do not apply.

Questions of nonlinear stability are frequently resolved by Lyapunov's direct method. Roughly speaking, a Lyapunov function $V$ is a nonnegative functional which is defined and continuously differentiable in a neighborhood of a equilibrium point $z_{0}$ and is uniquely minimized in that neighborhood by $z_{0}$. If

$$
\dot{V}(u)=\partial V(u) f(u) \leq 0
$$

in this neighborhood, then it follows that $z_{0}$ is a stable equilibrium point. Asymptotic stability can be deduced from conditions such as

$$
\dot{V}(u)<-\alpha V(u)
$$

for some $\alpha>0$. In certain cases, a Lyapunov functional satisfying (3.1) in a neighborhood of an equilibrium point of a system of ordinary differential equations is useful in the context of the associated reaction-diffusion system. For this purpose, we introduce the notion of $D$-diffusively convex Lyapunov functionals for reaction-diffusion systems.

Definition 3.3. Let $D$ be the matrix of diffusion coefficients for (1.2) and suppose that $M$ is a forward invariant rectangle (possibly unbounded) for (1.2). If $z_{0} \in M$ is an equilibrium point of $f$ we say that a nonnegative functional $V$ is a $D$-diffusively convex Lyapunov functional around $z_{0}$ provided that these conditions hold:

(i) There exists a $\xi>0$ such that $V \in C^{2}\left(M \cap B_{\xi}\left(z_{0}\right) ; \mathbb{R}^{+}\right)$.

(ii) There exist constants $r>0$ and $K>0$ such that

(iii) $V\left(z_{0}\right)=0$.

$$
V(u) \geq K \sum_{i=1}^{m}\left|u_{i}-z_{0_{i}}\right|^{r} \text { for } u \in B_{\xi}\left(z_{0}\right) \cap M
$$

(iv) The matrix $D \partial^{2} V(u)$ is positive semidefinite for $u \in B_{\xi}\left(z_{0}\right) \cap M$. (Here $\partial^{2} V(u)$ is the Hessian matrix of $V$.)

(v) $\partial V(u) f(u) \leq 0$ for $u \in B_{\xi}\left(z_{0}\right) \cap M$.

We remark that conditions (i)-(iii) and (v) are essentially those which define a Lyapunov functional for (1.1) around $z_{0}$ and that condition (iv) represents an additional strengthening 
of the concept. If the functional $V$ is separable; i.e.,

$$
V(u)=\sum_{i=1}^{n} V_{i}\left(u_{i}\right)
$$

then we may ensure (iv) by assuming that $V_{i}^{\prime \prime}\left(u_{i}\right) \geq 0$. In general, however, convexity of $V$ does not suffice for condition (iv). It is relatively straightforward to see that $D$-diffusively convex Lyapunov functionals guarantee the persistence of stability of equilibrium points. We have the following theorem.

THEOREM 3.4. Let $z_{0} \in M$ be an equilibrium point for the vector field $f$ where $M$ is a forward invariant set for the semilinear parabolic system (1.2). If there exists a $D$ diffusively convex Lyapunov functional $V$ for $f$ around $z_{0}$, then $z_{0}$ is a stable steady-state for (1.2) with respect to $M$. Moreover, if $V$ also satisfies (3.2), then $z_{0}$ is asymptotically stable with respect to $M$.

Proof. We choose $\eta>0$ so that the cube $C_{2 \eta}\left(z_{0}\right)$ is contained in $B_{\xi}\left(z_{0}\right)$, and we construct the truncated vector field $f\left[\eta, z_{0}\right]$ as in $(2.11),(2.12)$. If $v_{0}(x) \in C_{2 \eta}\left(z_{0}\right) \cap M$ for $x \in \Omega$ it is immediately verified that

$$
\partial V(v(x, t)) f(v(x, t))=\partial V(v(x, t)) f\left[\eta, z_{0}\right](v(x, t)) \leq 0 .
$$

If we multiply the $i^{\text {th }}$ component of $(2.12 \mathrm{a})$ by $\partial V(v) / \partial v_{i}$ we obtain

$$
\left(\partial V(v) / \partial v_{i}\right) \partial v_{i} / \partial t=d_{i}\left(\partial V(v) / \partial v_{i}\right) \Delta v_{i}+\left(\partial V(v) / \partial v_{i}\right) f_{i}\left[\eta, z_{0}\right](v) .
$$

If we integrate this expression on the space-time cylinder and sum the components, we observe that

$$
\begin{aligned}
\int_{\Omega} V(v(x, t)) d x & =-\int_{0}^{T} \int_{\Omega}(\nabla v)^{T} D \partial^{2} V(v) \nabla v d x d t \\
& +\int_{0}^{t} \int_{\Omega} \partial V(v) f\left[\eta, z_{0}\right](v) d x+\int_{\Omega} V\left(v_{0}(x)\right) d x
\end{aligned}
$$

Hence by virtue of conditions (iv) and (v) in Definition 3.3 we have

$$
\int_{\Omega} V(v(x, t)) d x \leq \int_{\Omega} V\left(v_{0}(x)\right) d x .
$$

Using (3.7) and the coercivity of $V$ we get

$$
\begin{aligned}
K\left[\sum_{i=1}^{m}\left\|v_{i}(\cdot, t)-z_{0, i}\right\|_{r, \Omega}\right] & \leq\left[\int_{\Omega} V(v(x, t)) d x\right]^{1 / r} \\
& \leq\left[\int_{\Omega} V\left(v_{0}(x)\right) d x\right]^{1 / r} \\
& \leq \rho\left(\sum_{i=1}^{m}\left\|v_{0_{i}}-z_{0_{i}}\right\|_{\infty, \Omega}\right)
\end{aligned}
$$


for some continuous $\rho$ with $\rho(0)=0$ and $\rho(s)>0$ for $s>0$. This will ensure $\infty-r$ stability, and from Theorem 2.16 we may conclude that $z_{0}$ is stable. Finally, in case (3.2) holds, we take $v_{0}$ sufficiently close to $z_{0}$ to guarantee our solution stays close to $z_{0}$ for all $t>0$. Then, one can obtain the estimate

$$
\int_{\Omega} V(v(x, t)) d x \leq e^{-\alpha t} \int_{\Omega} V\left(v_{0}(x)\right) d x
$$

and from this follows the asymptotic stability assertion.

In view of Theorem 2.37 one can be naturally lead to the attempt of using $D$-diffusively convex Lyapunov functions to analyze the stability of spatially non-homogeneous steadystate solutions. The following simple proposition squashes this endeavor for large classes of dynamical systems.

Proposition 3.10. Let $M$ be a forward invariant set for (1.2) and let $V(v)=$ $\sum_{i=1}^{m} V_{i}\left(v_{i}\right)$ be a nonnegative separable function which satisfies the defining hypotheses of Definition 3.3, except possibly (ii) and (iii), for all points of $M$. If $w=\left(w_{1}, \ldots, w_{m}\right)^{T} \in M$ is a solution to (2.35) then the following are true:

(i) If there exists $\alpha>0$ such that $V_{i}^{\prime \prime}\left(v_{i}\right)>\alpha$ for all $v=\left(v_{1}, \ldots, v_{m}\right)^{T} \in M$, then $f(w)=0$.

(ii) If $V(v)=\sum_{i=1}^{m} c_{i} v_{i}, \partial V(v) f(v) \leq 0$ and $M \subseteq \mathbb{R}_{+}^{m}$, then there exists a $k>0$ such that $y(x)=\sum_{i=1}^{m} c_{i} d_{i} w_{i}(x)=k$ for all $x \in \Omega$; i.e., $w(x)$ belongs to a closed bounded subset of the hyperplane $\left\{v \mid \Sigma c_{i} d_{i} v_{i}=k\right\} \cap \mathbb{R}_{+}^{m}$.

Proof. In the first case we multiply the $i^{\text {th }}$ component of (2.35a) by $V_{i}^{\prime}\left(w_{i}\right)$ to obtain

$$
-d_{i} V_{i}^{\prime}\left(w_{i}\right) \Delta w_{i}=V_{i}^{\prime}\left(w_{i}\right) f_{i}(w)
$$

If we sum these terms and integrate on $\Omega$ we have

$$
\sum_{i=1}^{m} d_{i} \int_{\Omega} V_{i}^{\prime \prime}\left(w_{i}\right)\left|\nabla w_{i}\right|^{2} d x=\sum_{i=1}^{n} \int_{\Omega} V_{i}^{\prime}\left(w_{i}\right) f_{i}(w) d x \leq 0 .
$$

Consequently,

$$
\sum_{i=1}^{m} \alpha d_{i} \int_{\Omega}\left|\nabla w_{i}\right|^{2} d x=0
$$

and we may conclude that each $w_{i}$ is a constant. Therefore, because $w=\left(w_{1} \ldots w_{n}\right)^{T}$ is a solution to $(2.35)$, we must have $f_{i}(w)=0$. If we follow the same train of reasoning for the second case then we observe that $-\Delta\left(\Sigma c_{i} d_{i} w_{i}\right) \leq 0$. The fact that $M$ is required to lie in $\mathbb{R}_{+}^{m}$ implies that $\Sigma d_{i} w_{i} \geq 0$, and hence we conclude from maximum principles that $\nabla\left(\Sigma c_{i} d_{i} w_{i}\right)$ vanishes and $\Sigma c_{i} d_{i} w_{i}(x)=k$ for some constant $k \geq 0$. Thus $w(x)$ lies in the hyperplane $\left\{v \mid \Sigma c_{i} d_{i} v_{i}=k\right\}$. The continuity of $y$ implies that its range is closed and bounded. 
As a closing remark for this section, we point out that additional treatment of Lyapunov theory in the context of reaction-diffusion systems can be found in [20].

\section{Applications.}

We begin with the consideration of the two component system

$$
\begin{array}{ll}
\partial u / \partial t-d_{1} \Delta u=-f(u, v) & \text { on } \Omega \times(0, \infty), \\
\partial v / \partial t-d_{2} \Delta v=f(u, v) & \text { on } \Omega \times(0, \infty), \\
\partial u / \partial \mathbf{n}=\partial v / \partial \mathbf{n}=0 & \text { on } \partial \Omega \times(0, \infty), \\
u(\cdot, 0)=u_{0}(\cdot), v(\cdot, 0)=v_{0}(\cdot) & \text { on } \Omega,
\end{array}
$$

where $f \in C^{2}\left(\mathbb{R}_{+}^{2} ; \mathbb{R}_{+}\right)$and $f(0, v)=0$ for all $v \in \mathbb{R}_{+}$. Here we assume that the initial data $u_{0}, v_{0}$ are continuous and nonnegative on $\bar{\Omega}$. It may be surprising that questions concerning the global existence of solutions to this system remain open. If the nonlinearity $f$ is polynomially bounded, then it is known [10] that solutions to (4.1) exist in the large and remain uniformly bounded in the $L_{\infty}(\Omega)$ norm. Analogous results, [8], have also have been obtained in case the nonlinearity is of the form

$$
f(u, v)=u \varphi(v)
$$

where $\varphi$ need not be polynomially bounded but is required to grow less than exponentially; e.g., $\varphi(v)=e^{\sqrt{v}}$.

We are able to establish a simple result concerning the stability of the steady state $(0, \tilde{v})$ for $(4.1)$.

Proposition 4.3. If $\tilde{v} \geq 0$ then the constant solution $(u, v)=(0, \tilde{v})$ is a stable equilibrium point for (4.1) with respect to $\mathbb{R}_{+}^{2}$.

Proof. By assumption $f(0, \tilde{v})=0$, and hence $(0, \tilde{v})$ is a steady-state solution of the system. In the case in which $\tilde{v}=0$ the result follows by noting that $\mathbb{R}_{+}^{2}$ is an invariant $m$-cube for the system, that $\infty$-1 stability follows from integrating each equation on the space-time cylinder and adding them to obtain the conservation law

$$
\int_{\Omega}(u(x, t)+v(x, t)) d x=\int_{\Omega}\left(u_{0}(x)+v_{0}(x)\right) d x,
$$

and that $V=u+v$ defines a $D$-diffusively convex Lyapunov functional around $(0,0)$ with respect to $\mathbb{R}_{+}^{2}$. Now suppose that $\tilde{v}>0$ and let $0<\varepsilon<\tilde{v}$. Maximum principles demonstrate that solutions which initially lie in $M_{\varepsilon}=\{(u, v) \mid u \geq 0, v \geq \tilde{v}-\varepsilon\}$ remain so. The conservation law

$$
\int_{\Omega}(u(x, t)+v(x, t)-(\tilde{v}-\varepsilon)) d x=\int_{\Omega}\left(u_{0}(x)+v_{0}(x)-(\tilde{v}-\varepsilon)\right) d x
$$

follows as before. Thus, if the initial data are close to $(0, \tilde{v}-\varepsilon)$ in the $L^{\infty}$ norm, then the solution remains close in the $L^{1}$ norm. Also, $V=u+v-(\tilde{v}-\varepsilon)$ defines a $D$-diffusively convex Lyapunov functional around $(0, \tilde{v}-\varepsilon)$ with respect to $M_{\varepsilon}$. Consequently, $\infty-1$ 
stability implies uniform stability with respect to $M_{\varepsilon}$. Therefore, it follows that solutions in $\mathbb{R}_{+}^{m}$ can be made to remain uniformly close to $(0, \tilde{v})$.

We hope that we do not belabor the issue by pointing out that the system

$$
\begin{aligned}
& \partial u / \partial t=d_{1} \Delta u-u e^{k v^{\gamma}} \\
& \partial v / \partial t=d_{2} \Delta v+u e^{k v^{\gamma}}
\end{aligned}
$$

for example, with $\partial u / \partial \mathbf{n}=\partial v / \partial \mathbf{n}=0$ on $\partial \Omega$ and any $\gamma \geq 1$ satisfies the hypotheses and hence admits $(0, \tilde{v})$ as a stable solution, with respect to $M$ as above, whenever $\tilde{v} \geq 0$.

We now focus on a general class of diffusive Lotka-Volterra systems. Typically LotkaVolterra systems feature quadratic nonlinearities. They are intended to describe the species interaction among $m$-species ecological systems. Here, we follow the development of Leung [13] and consider systems of the form

$$
\begin{aligned}
\partial u / \partial t & =D \Delta u+U(e+P u) & & \text { on } \Omega \times(0, \infty), \\
\partial u / \partial \mathbf{n} & =0 & & \text { on } \partial \Omega \times(0, \infty), \\
u(\cdot, 0) & =u_{0}(\cdot), & & \text { on } \Omega,
\end{aligned}
$$

where $U=\operatorname{diag}\left\{u_{1}, \ldots, u_{m}\right\}, e=\left(e_{1}, \ldots, e_{m}\right)^{T}$ is a constant vector, and $P=\left(p_{i j}\right)$ is an $m \times m$ matrix with constant entries. We assume that the following conditions are satisfied. $(\mathrm{L}-\mathrm{V})_{1}$ There is a vector $q=\left(q_{1}, \ldots, q_{m}\right)^{T}$, with each $q_{i}>0$, that solves the linear system

$$
e+P q=0
$$

$(\mathrm{L}-\mathrm{V})_{2} \quad$ For each $q$ satisfying (4.7) there is a diagonal matrix $A=\operatorname{diag}\left\{a_{1}, \ldots, a_{m}\right\}$, with each $a_{i}>0$, such that for all $w \in \mathbb{R}^{m}$,

$$
(A w)^{T} P w=\sum_{i, j=1}^{m} a_{i} w_{i} p_{i j} w_{j} \leq 0
$$

Condition $(\mathrm{L}-\mathrm{V})_{1}$ guarantees the existence of a steady state with positive components. However, we have made no assumptions concerning the nonsingularity of the matrix $P$. Indeed, many Lotka-Volterra systems feature a multiplicity of positive steady states. The nonnegativity of the quadratic form (4.8) translates as weighted conservation of the interaction between the species of the system. Leung refers to this condition as admissibility.

The next lemma asserts that a well-known Lyapunov function for (4.6) provides a $D$-diffusively convex Lyapunov structure.

LEMmA 4.9. There exists $\xi>0$ such that the function $V$ on $\mathbb{R}_{+}^{m} \cap B_{\xi}(q)$ defined by

$$
V(v)=\sum_{i=1}^{m} V_{i}\left(v_{i}\right)=\sum_{i=1}^{m}\left(a_{i}\left(u_{i}-q_{i}\right)-a_{i} q_{i} \log \left(u_{i} / q_{i}\right)\right)
$$

is D-diffusively convex on $\mathbb{R}_{+}^{m} \cap B_{\xi}(q)$. 
Proof. If $B_{\xi}(q)$ does not intersect the coordinate hyperplanes of $\mathbb{R}_{+}^{m}$, then it is clear that $V$ is continuously differentiable and nonnegative on $B_{\xi}(q)$. Moreover, it is clear that $V(q)=0$, and a careful analysis will reveal that $K>0, \xi>0$ and $r>0$ may be chosen so that hypothesis (ii) of Definition 3.3 holds. We observe that if $u \in \mathbb{R}_{+}^{m}$, then

$$
\partial V(u) f(u)=(A(u-q))^{T} P(u-q)=\sum_{i, j=1}^{m} a_{i}\left(u_{i}-q_{i}\right) P_{i j}\left(u_{j}-q_{j}\right) \leq 0 .
$$

The separability of $V$ and the observation that $V_{i}^{\prime \prime}\left(v_{i}\right)=a_{i} q_{i} / v_{i}$ complete the proof.

We immediately have the following result.

Proposition 4.12. If $(L-V)_{1}$ and $(L-V)_{2}$ are satisfied, then the steady-state solution $q=\left(q_{1}, \ldots, q_{m}\right)^{T}$ is stable. Moreover the semilinear elliptic system

$$
\begin{array}{llrl}
-D \Delta w & =W(e+P w) & & \text { on } \Omega \\
\partial w / \partial \mathbf{n} & =0 & & \text { on } \partial \Omega,
\end{array}
$$

where $W=\operatorname{diag}\left\{w_{1}, \ldots, w_{m}\right\}$, has no spatially nonhomogeneous positive solutions.

Proof. Because $\mathbb{R}_{+}^{m}$ is an invariant $m$-cube for solutions to (4.6), Lemma 4.9 and Theorem 3.4 establish the first assertion. To establish the second assertion, we let $M_{1}$ be an $m$-cube which contains both $w \in \mathbb{R}_{+}^{m}$ and $q \in \mathbb{R}_{+}^{m}$ and does not intersect the coordinate hyperplanes of $\mathbb{R}_{+}^{M}$, and we let $M_{2}$ be a second $m$-cube which contains $M_{1}$ and also does not intersect the coordinate hyperplanes. Now let $\varphi \in C^{\infty}\left(\mathbb{R}^{m} ; \mathbb{R}_{+}\right)$be such that $\varphi(u)=1$ if $u \in M_{1}$ and $\varphi(u)=0$ for $u \in \mathbb{R}^{m} \backslash M_{2}$. From application of Proposition 3.10, part (i), to the truncated system

$$
\begin{array}{llrl}
-D \Delta w & =\varphi(w) W(e+P w) & & \text { on } \Omega \\
\partial w / \partial \mathbf{n}=0 & & \text { on } \partial \Omega,
\end{array}
$$

the remaining assertion follows directly.

We mention that for $n \geq 3$ the question of global existence for (4.6) is in general unresolved. For spatial dimension one, global existence and uniform boundedness for solutions may be established by applying results in [16], and for $n=2$ we are at least assured the existence of longtime solutions; see [17]. We mention this to underscore the point that global well-posedness theory for reaction-diffusion systems remains incomplete.

Differential equations which describe the dispersion and reaction of $m$ chemical species are generally of the form

$$
\partial u / \partial t=D \Delta u+f(u)
$$

where the $i^{\text {th }}$ component of the dependent variable $u=\left(u_{1}, \ldots, u_{m}\right)^{T}$ represents the concentration density of the $i^{\text {th }}$ chemical species. The vector field $f=\left(f_{i}\right)_{i=1}^{m}$ is assumed to be in each component a polynomial function of the components of $u$ and is intended to model the chemical reaction kinetics. Gröger, in his study of dissipative chemical reactions, [7], introduced the following hypothesis. 
(G) There exists a vector $e=\left(e_{1}, \ldots, e_{m}\right)^{T}$ with each $e_{i}>0$ such that $f(e)=0$ and

$$
\sum_{i=1}^{m} f_{i}(u) \log \left(u_{i} / e_{i}\right) \leq 0
$$

Furthermore, the quantity $\sum_{i=1}^{m} f_{i}(u) \log \left(u_{i} / e_{i}\right)$ is known to have the physical interpretation of being a suitably scaled rate of chemical dissipation, and work on the mathematical theory of reaction networks, [11], confirms that many nontrivial systems satisfy this hypothesis. If the chemical species are required to remain confined to a reaction vessel for all time the appropriate boundary conditions are given by

$$
\partial u / \partial \mathbf{n}=0 \quad \text { on } \partial \Omega \times(0, \infty) .
$$

Finally, a condition of the form

$$
f_{i}(u) \geq 0 \quad \text { for all } u \in \mathbb{R}_{+}^{m} \text { with } u_{i}=0
$$

together with the maximum principle ensures that $\mathbb{R}_{+}^{m}$ is a forward invariant set for (4.15). We have the following proposition.

Proposition 4.19. We consider (4.15) together with the boundary conditions (4.16). If all the conditions describing a dissipative chemical reaction outlined above hold, then the steady state $u=e$ is uniformly stable. Moreover, the elliptic system

$$
\begin{aligned}
-D \Delta w & =f(w) & & \text { on } \Omega \\
\partial w / \partial \mathbf{n} & =0 & & \text { on } \partial \Omega
\end{aligned}
$$

has no spatially inhomogeneous positive solutions.

Proof. We define

$$
V(u)=\sum_{i=1}^{m} V_{i}(u)=\sum_{i=1}^{m}\left(u_{i} \log \left(u / e_{i}\right)-u_{i}+e_{i}\right)
$$

and verify that all conditions of Definition 3.3 hold locally about $e$. Consequently, Theorem 3.4 implies that $e$ is uniformly stable. An argument analogous to the one of Proposition 4.12 ensures the nonexistence of positive spatially inhomogeneous steady-states.

The comments concerning the global well-posedness and boundedness of solutions to Lotka-Volterra systems also apply to this class of dissipative chemical systems.

In addition to satisfying $f(0)=0$ and a condition of the form (4.18), many reactiondiffusion systems satisfy a linear balancing condition of the form:

(B) There exist positive constants $c_{i}$ for $i=$ to $m$ so that for all $n \in \mathbb{R}_{+}^{m}$,

$$
\sum_{i=1}^{m} c_{i} f_{i}(u)=0
$$


In this case, an obvious generalization of Proposition 4.3 dictates the stability of the zero solution.

\section{Further generalizations and concluding remarks.}

Our results tend to support the general hypothesis that the addition of diffusion to systems of ordinary differential equations which have $D$-diffusively convex Lyapunov functions does not create exotic spatial or temporal phenomena which did not originally exist. If indeed this is the case, then the presence of diffusion in these systems is irrelevant to their long-term dynamics, and any spatial phenomena produced by diffusion must be of a transient nature.

We need not have limited our consideration to diffusion mechanisms of the form $D \Delta u$. We could have allowed operators of the form

$$
\sum_{j, k=1}^{n} \frac{\partial}{\partial x_{k}}\left(d_{j k}^{i}(x, t) \frac{\partial u_{i}}{\partial x_{j}}\right)
$$

in each component. In this case it is necessary to assume uniformly strong ellipticity along with smoothness conditions on coefficients and some conditions on the derivatives of the coefficients. In general, the arguments could become quite technical, but should be tractable. We leave the details to the interested reader. Numerical experiments, [4], with two component systems which model exothermic chemical reactions indicate that quasilinear diffusivities do have an effect on the intermediate dynamics of the systems.

The necessity that our forward invariant set $M$ be an $m$-cube described by (2.4) is purely a consequence of assuming distinct diffusion coefficients and in no way actually enters into the preceding analysis. Other types of geometries can arise in situations where some of the diffusion coefficients are equal. As a simple example, consider a threecomponent model of the form:

$$
\begin{array}{lc}
\partial u / \partial t-\Delta u=-\alpha_{1} f(u, v, w) & \text { on } \Omega \times(0, \infty), \\
\partial v / \partial t-\Delta v=-\alpha_{2} f(u, v, w) & \text { on } \Omega \times(0, \infty), \\
\partial w / \partial t-d \Delta w=f(u, v, w) & \text { on } \Omega \times(0, \infty), \\
\partial u / \partial \mathbf{n}=\partial v / \partial \mathbf{n}=\partial w / \partial \mathbf{n}=0 & \text { on } \partial \Omega \times(0, \infty), \\
u(\cdot, 0)=u_{0}, v(\cdot, 0)=v_{0}, w(\cdot, 0)=w_{0} & \text { on } \Omega,
\end{array}
$$

where $f \in C^{2}\left(\mathbb{R}_{+}^{3} ; \mathbb{R}_{+}\right) ; f(0, v, w)=f(u, 0, w)=0$ for all $u, v, w \in \mathbb{R}_{+} ; \alpha_{1}, \alpha_{2}, d>0$; and the initial data $u_{0}, v_{0}, w_{0}$ are continuous and nonnegative on $\bar{\Omega}$. By the maximum principle, it follows that $\min _{\Omega}\left\{\alpha_{1} v_{0}-\alpha_{2} u_{0}\right\} \leq \alpha_{1} v-\alpha_{2} u \leq \max _{\Omega}\left\{\alpha_{1} v_{0}-\alpha_{2} u_{0}\right\}$ and $w \geq \min _{\Omega} w_{0}$. Consequently, if $z_{1}, z_{3} \geq 0$, then the set

$$
M_{1}=\left\{(u, v, w) \mid \alpha_{1} v-\alpha_{2} u \leq-\alpha_{2} z_{1}, v \geq 0, w \geq z_{3}\right\}
$$

is a forward invariant set for (5.1), and if $z_{2}, z_{3} \geq 0$, then the set

$$
M_{2}=\left\{(u, v, w) \mid u \geq 0, \alpha_{1} v-\alpha_{2} u \geq \alpha_{1} z_{2}, w \geq z_{3}\right\}
$$


is a forward invariant set for (5.1). Now, in a manner similar to the proof of Proposition 4.3 , one can show that any point $\left(z_{1}, 0, z_{3}\right)$ with $z_{1}, z_{3} \geq 0$ is stable with respect to $M_{1}$, and any point $\left(0, z_{2}, z_{3}\right)$ with $z_{2}, z_{3} \geq 0$ is stable with respect to $M_{2}$. One can then continue to argue as in the proof of Proposition 4.3 that such equilibrium points are stable with respect to $\mathbb{R}_{+}^{3}$.

\section{REFERENCES}

[1] N. Alikakos, An application of the invariance principle to reaction-diffusion equations, J. Differential Equations 33 (1979), pp. 201-225.

[2] H. Amann, Existence and regularity for semilinear parabolic evolution equations, Annali Scoula Nosmale Superiore - Pisa, Serie IV, Vol. IX (1984), pp. 593-676.

[3] J.F.G. Auchmuty, Qualitative effects of diffusion in chemical systems, Lectures on Mathematics in the Life Sciences, 10 (1978), pp. 49-99.

[4] W. FitzGibBon And C. MARTin, The longtime behavior of solutions to a quasilinear combustion model, J. Nonlinear Analysis, Vol 19 No. 10 (1992), pp. 947-961.

[5] W. Fitzgibbon, J. Morgan and R. Sanders, Global existence and boundedness for a class of inhomogeneous parabolic equations, J. Nonlinear Analysis, Vol. 19, No. 9 (1992), pp. 885-899.

[6] W. Fitzgibbon, J. Morgan and S. Waggoner, Weakly coupled semilinear parabolic evolution systems, Annali di Matematica Pure et Applicata, Vol. CLXI (1992), pp. 213-229.

[7] K. Gröger, On the existence of steady-states of certain reaction-diffusion systems, Arch. Rat. Mech. Anal. (1986), pp. 297-306.

[8] A. HaRAuX AND A. YoukANA, On a result of $K$. Masuda concerning reaction-diffusion equations, Tohoku Math. J., 40 (1988), pp. 158-183.

[9] D. Henry, Geometric Theory of Semilinear Parabolic Equations, Lecture Notes in Mathematics, 840, Springer-Verlag, Berlin, 1981.

[10] S. Hollis, R. MARTIN AND M. Pierre, Global existence and boundedness in reaction-diffusion systems, SIAM J. Math. Anal., 18 (1987), pp. 744-761.

[11] F. Horn and R. Jackson, General mass action kinetics, Arch. Rat. Mech. and Anal., 47 (1972), pp. 81-116.

[12] O. Ladyženskaja, V. Solonnikov and N. Uralćeva, Linear and Quasilinear Equations of Parabolic Type, AMS Trans., Vol. 23, Amer. Math. Soc., Providence, R.I., 1968.

[13] A. Leung, Systems of Nonlinear Partial Differential Equations, Kluwer Academic Publ., Boston, 1989.

[14] R. Martin And M. PierRe, Nonlinear reaction diffusion systems, in Nonlinear Equations in the Applied Sciences, W.F. Ames and C. Rogers, editors, Academic Press, 1990.

[15] H. Matano, Asymptotic behavior and stability of solutions of semilinear diffusion equations, Publications of the Research Institute for Mathematical Science, 15, (1979), pp. 401-454.

[16] J. Morgan, Boundedness and decay results for reaction-diffusion systems, SIAM J. Math. Anal., 21 (1990), pp. 1172-1181.

[17] J. Morgan, Global existence for a class of quasilinear reaction-diffusion systems, Preprint.

[18] J. Murray, Mathematical Biology, Springer-Verlag, Berlin, 1989.

[19] M. Pierre AND D. Schmidt, Blow-up in reaction-diffusion systems with dissipation of mass, Les prépublications de l'Institut Élie Cartan, 95/No.32, Université Henri Poincaré Nancy I.

[20] R. Redheffer, R. Redlinger and W. Walter, A theorem of LaSalle-Lyapunov type for parabolic systems, SIAM J. Math. Anal., 19 (1988), pp. 121-132.

[21] J. Smoller, Shock Waves and Reaction-Diffusion Equations, Springer-Verlag, Berlin, 1984.

[22] H. STEWART, Generation of analytic semigroups by strongly elliptic operators under general boundary conditions, Trans. Amer. Math. Soc., (1980), pp. 299-310. 